\title{
Author Correction: Image-guided radiotherapy platform using single nodule conditional lung cancer mouse models
}

Grit S. Herter-Sprie, Houari Korideck, Camilla L. Christensen, Jan M. Herter, Kevin Rhee, Ross I. Berbeco, David G. Bennett, Esra A. Akbay, David Kozono, Raymond H. Mak, G. Mike Makrigiorgos, Alec C. Kimmelman \& Kwok-Kin Wong

Correction to: Nature Communications https://doi.org/10.1038/ncomms6870, published online 18 December 2014.

The original version of this Article contained an error in Fig. 4, panel a. The representative image for the 12 hour time point in the KL group (second from the right, middle raw) was inadvertently replaced with an inverted duplicate of the image for the 24 hour time point in the same group (the last image on the right, middle raw). The correct version of Fig. 4a is:

a

8 Gy IR

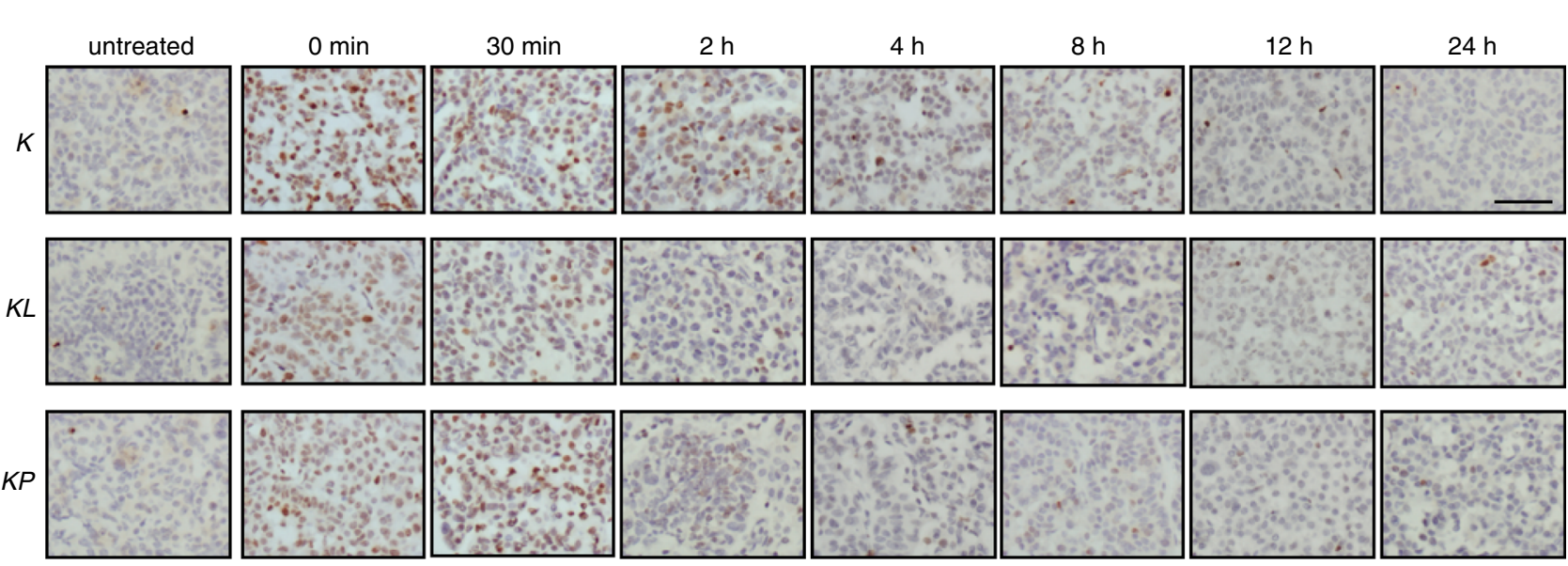

It replaces the previous incorrect version, which is: 


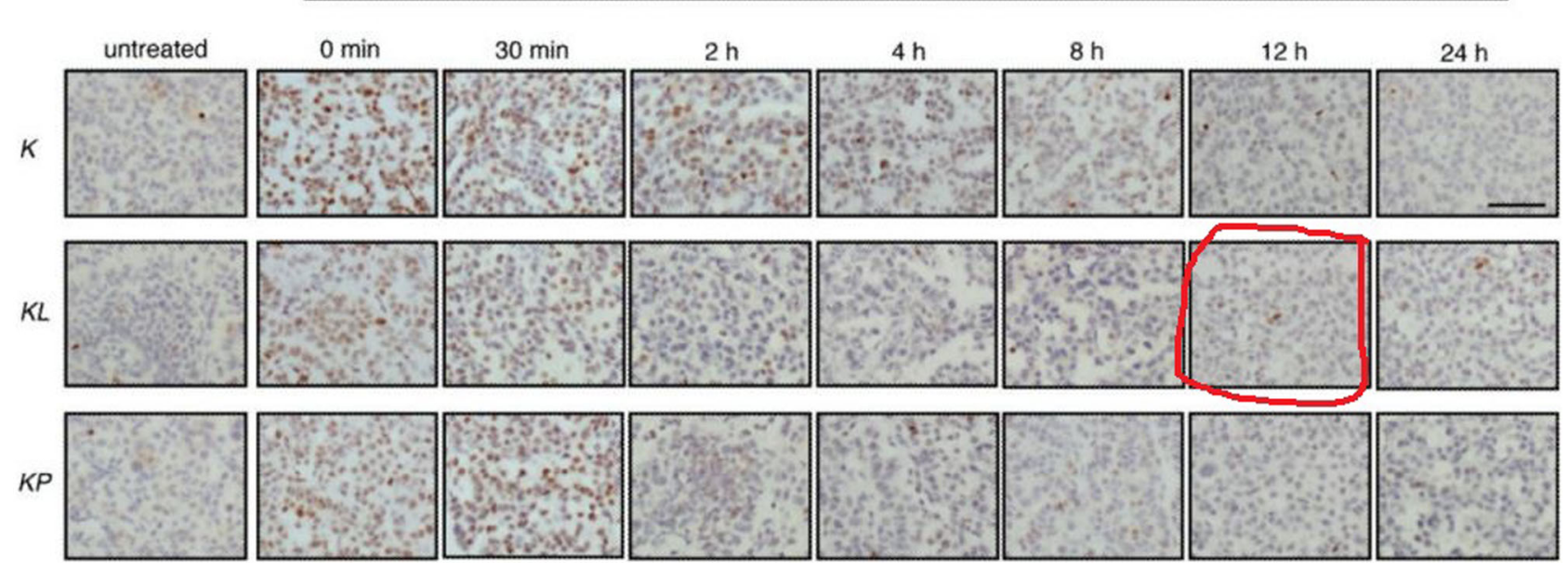

This error is not corrected in the PDF nor HTML versions of the Article. The original conclusions are not affected by this change.

Published online: 09 April 2020

(C) The Author(s), under exclusive licence to Springer Nature Limited 2020 